\title{
German Particle Verbs and Pleonastic Prepositions
}

\author{
Ines Rehbein \\ NCLT \\ School of Computing, DCU, \\ Dublin, Ireland \\ irehbein@computing.dcu.ie
}

\section{Introduction}

The subject of this paper are German particle verbs with pleonastic prepositions (5). In German there are nine two-way prepositions which can either govern the accusative or the dative: an, auf, hinter, in, neben, über, unter, vor and zwischen. The difference in case assignment also causes a different interpretation of the semantics of the prepositional phrase: if the preposition governs the dative it expresses a locative relation (1), while the accusative goes together with a directional interpretation (2).

\author{
Josef van Genabith \\ NCLT, \\ School of Computing, DCU, \\ Dublin, Ireland \\ josef@computing.dcu.ie
}

(1) Das Bild hängt [PP an der Wand].

Det Picture hang-3Sg [PP on - dir Det $_{d a t}$ wall].

'The picture hangs on the wall.'

(2) Sie hängt das Bild [PP an die Wand].

She hang-3Sg Det picture [PP onto $+d i r$ Det $_{a c c}$ wall].

'She hangs the picture on the wall.'

The two-way prepositions combined as prefixes with a verb form the so-called particle verbs (also called separable prefix verbs). The particles implicitly include directional information and can change the aspectual mode and argument structure of their base verbs. Particle verbs can be differentiated according to whether they allow for a pleonastic combination with the particle in question and the resulting syntactic and semantic effects.

Olsen (1998) refers to this phenomenon as the Pleonastic Directional, where the verb particle already saturates the directional requirement of the verb and therefore there should be no need for a further preposition offering the same directional information. However, example (5) shows that pleonastic directionals can in fact occur with directional PPs, while in (3) the main verb (without particle) combines with a directional PP and in (4) only the particle verb is used.

(3) Sie steigt [PP in das Auto].

She climb-3SG [PP into $+D I R$ Det car].

'She gets into the car.'

(4) Sie steigt ein.

She climb-3SG Part $+D I R$.

'She gets in.'

(5) Sie steigt [PP in das Auto] ein.

She gets $\left[\mathrm{PP}\right.$ into $_{+D I R}$ Det car] Part $+D I R$.

'She gets into the car.'

The problem is that it is not clear what licenses 
the directional preposition in cases such as (5) and why it is not supressed by the verb particle.

The base verb in (3) licenses a directional PP, which is part of the argument structure of the verb. If there is a verb particle which saturates this directional requirement (4), then the realisation of the PP is optional. Wunderlich (1983) argues that particle verbs require a stereotype or contextually given object equal to the internal argument of the prepositional relation, which can be reconstructed from the context and therefore can be omitted.

If the directional information is already represented by the particle, then the question arises what licenses the directional PP. It could be argued that the particle should suppress a directional PP or, conversely that the directional PP should suppress the verb particle. The question which of the two is selected first, the particle verb or the preposition, is discussed controversially. In a speakeroriented view the particle verb will be selected first, while the theory of linear sentence processing claims that the particle, which is only encountered at the end of the sentence, should be omitted.

Particle verbs with pleonastic PPs exhibit another interesting property: some of them only allow for pleonastic prepositions governing dative PPs while others trigger the accusative, and some particle verbs can even go together with both cases. The underlying reasons for those case preferences are not completely clear.

It is obvious that there are certain verb classes whose semantics seem to influence the case assigned by the preposition. This is strongly connected with the influence of directional information concerning the case preference of the particle verb. Particle verbs which express directional information trigger PPs in the accusative, while particle verbs whose semantics contain no directional component never combine with an accusative PP. But why are there also particle verbs which are able to combine with both cases?

The aim of this paper is to give an explanation for this phenomenon, based on data gained through corpus research. Section 2 describes characteristic features of spatial prepositions and particle verbs. Section 3 presents a novel corpusbased typology of verb classes triggering different case for pleonastic prepositions, accounting for regularities in their observed behaviour. Section 4 provides a novel account of particle verbs with their pleonastic prepositions using the framework of Lexical Functional Grammar (Bresnan, 2000). The last section summarizes the main results established in this paper.

\section{Characteristic Features of Particle Verbs and Spatial Prepositions}

Spatial prepositions are binary relations between two entities, where one of the entities is located with respect to a region defined by the second entity, specified through the preposition. The meaning of a two-way preposition depends on the case of the PP: if it is in the dative, its reading will be interpreted as a static, non-directional localisation, while the accusative triggers a directional interpretation. In the latter case the preposition implies a change of location of the theme referent from an unspecified region into the neighbouring region of the relatum (Witt, 1998).

In this paper we only deal with spatial prepositions, ignoring lexicalised prepositions without semantic content, as in (6):

(6) Sie wartet auf den Bus.

She wait-3Sg for Det bus.

'She is waiting for the bus.'

Dalrymple (2001) refers to (6) as idiosyncratic case, because the lexical form of the preposition is not related to the semantic role of the argument, while oblique arguments which are marked according to the semantic role of the argument are assigned semantic case. Particle verbs formed by two-way prepositions always have a semantic content.

The semantics of verb particles basing on spatial prepositions is equivalent to the semantics of the prepositions. They are also binary, but the internal argument of the relation is not explicitly expressed in the argument structure of the complex verb, but can be omitted (see examples (3) and (4)). The semantics of the particle is integrated into the semantics of the base verb which requires a directional complement.

In example (5) both particle verb and pleonastic PP occur together. Here the PP specifies the implicit reference object of the particle verb, and its relation of localisation is congruent with the directional semantics of the particle.

These characteristic features of particle verbs and spatial prepositions are constitutive for the classification into semantic verb classes given in Section 3. 


\section{Corpus-Based Classification of Particle Verbs with Pleonastic Prepositions}

The classification of particle verbs with pleonastic prepositions into semantic verb classes is based on the proposals by Witt (1998) extented by the results of our own corpus research. ${ }^{1}$ Witt's classification only considers particle verbs with the particle ein-. He divides them into three major groups: compositional formations, regular formations and non-compositional formations, which can be further subclassified into more fine-grained subclasses (Figure 1).

\section{Compositional Formations}

(a) Verb bases are causative Verbs of Localisation

(b) Verb bases are (static) Verbs of Localisation

(c) Verb bases are intransitive Verbs of Motion

(d) Verb bases are transitive Verbs of Motion (Transport Verbs)

2. Regular Formations

(a) Verb Bases are Activity-Verbs

(b) Verb Bases are 'eingravieren (to engrave)'-Verbs

3. Non-Compositional Formations: Extensions of Meaning

(a) Verb Bases are ein-Verbs with the meaning: 'downward, inward, into itself'

(b) Verb Bases are ein-Verbs with the meaning: 'to enclose something'

Figure 1: Witt's (1998) classification of particle verbs with ein-

In contrast to Witt, our classification includes all two-way prepositions as verb particles. As we are trying to explain the behaviour of particle verbs in regard to their ability to combine with pleonastic PPs, we divide the corpus data into the following groups: particle verbs licensing pleonastic PPs in the accusative only (Group A), particle verbs licensing pleonastic PPs in the dative only (Group B) and particle verbs which are able to govern either accusative or dative PPs (Group C).

Each of these groups can be divided into a number of subgroups, formed by different semantic verb types. Figure 2 gives an overview of our classification scheme.

\footnotetext{
${ }^{1}$ The corpora used for the research are the text basis of the Digital Dictionary of German Language (DWDS) (http://www.dwds.de/textbasis) and the corpora of the Institute of German Language (IDS) in Mannheim (http://www.ids-mannheim.de/cosmas2).
}

1. Group A (combine only with accusative PPs)
(a) Verb bases are (static) Verbs of Localisation
(b) Verb bases are intransitive Verbs of Motion
(c) Verb bases are transitive Verbs of Motion (Transport Verbs)
(d) Verb bases are Verbs of Perception
(e) Verb bases express a Change of State

2. Group B (combine only with dative PPs)
(a) Verb bases are (static) Verbs of Localisation
(b) Verb bases are intransitive Verbs of Motion
(c) Verb bases are (causative) Verbs of Position

3. Group $\mathbf{C}$ (combine with accusative and dative PPs)
(a) Verb bases are intransitive Verbs of Motion
(b) Verb bases are transitive Verbs of Motion (Transport Verbs)
(c) Verb bases express an Inclusion into an Environment, Institution or Abstract Area (d) Verb bases express Effects of Action
(eingravieren-Verbs)

Figure 2: Classification of particle verbs with twoway prepositions

\subsection{Group A}

The verbs in Group A licence PPs in the accusative and have a directional reading. Group A includes Verbs of Motion, Verbs of Localisation, Transport Verbs, and two further subgroups: verbs whose meaning can be interpreted as a Direction of Perception and verbs which express the Localisation of a Change of State.

Verbs of Motion include einfahren 'to drive into' or aufspringen 'to jump on' and can be defined as follows: there is an $\mathrm{X}$ which undergoes a change of location, whereby $X$ is in a particular manner of motion and moves in the specified direction into a not further specified neighbour region which is defined through the relatum.

Verbs of Localisation licencing PPs in the accusative are rather rare. Only one example is attested in the corpus: einmünden 'to discharge into'. Here an $\mathrm{X}$ is described, which can be localised relativ to a $\mathrm{Y}$ in a particular direction. The rarity of those verbs is probably due to the more static character of localisation, which contradicts the implicit directional reading of the accusative case marking.

Transport Verbs such as eingießen 'to pour in', einführen 'to insert' and also verbs with more metaphorical readings like einbinden (in die Kon- 
ventionen einbinden, 'to weave sth into social conventions'), can be defined in the following way: there is an $X$ which causes a change of location for a $\mathrm{Y}$, whereby $\mathrm{Y}$ is set into a particular manner of motion and is moved in a specified direction.

Direction of Perception verbs include einfühlen 'to empathise', einsehen 'to see' or einhören 'to listen'.

Localisation of a Change of State verbs include aufblähen 'to bloat', aufheizen 'to heat up', angleichen 'to conform to something' or aufrunden 'to round up'. Here the particle expresses the direction to the changed, new state.

All particle verbs in Group A can be interpreted as having a directional reading.

\subsection{Group B}

Particle Verbs in Group B licence pleonastic PPs in the dative. They can be divided into the following subgroups: Verbs of Localisation, Verbs of Movement and Position Verbs.

Verbs of Localisation also occur in Group A, but here they have a static, non-directional interpretation of localisation. Examples for this are verbs like einquartieren 'to quarter', anstehen 'to queue', aufliegen 'to bear on' or zwischenlagern 'to store temporarily'.

\section{(7) an a ART $_{\text {stehen }}$ \\ (PART + to stand $\rightarrow$ to queue).}

More formally they can be described as follows: There is an $\mathrm{X}$ which is in a particular state (e.g. in the state of standing) and can be localised in a specific relation to a reference object.

Verbs of Motion include vorfahren 'to drive up' or hinterherhecheln 'to pand after someone'. They can be defined as follows: there is an $\mathrm{X}$ which undergoes a change of location, whereby $\mathrm{X}$ is in a particular manner of motion, moving into the specified direction relative to the position of the relatum. These verbs clearly include an implicit direction, but in comparison to the Verbs of Motion in Group A their reading allows for the possibility that $\mathrm{X}$ is already in the same region as the relatum, while the verbs in Group A describe the intrusion of an $\mathrm{X}$ from the outside into a not further specified neighbour region.

Verbs of Position include aufstellen 'to array', aufbahren 'to lay out' or hinterlegen 'to deposit'.
The definition states that there is an $\mathrm{X}$ which causes a $\mathrm{Y}$ to change its position, whereby $\mathrm{Y}$ is in a particular manner of motion, moving into a specified direction. The focus hereby is not on the movement but on the result of the event.

The verbs in Group B normaly have a nondirectional, static interpretation, but they may also allow for a directional interpretation, if theme referent and relatum are both positioned in the same specified region (8).

(8) Sie stellt die Leiter [PP auf dem Podest] auf. She put-3-Sg Det ladder [PP on Det platform] Part. 'She puts the ladder up on the platform.'

Here it is not the direction of a motion which is described by the particle (the ladder may already have been lying on the platform), but a change of the orientation of the referent in relation to the relatum (the ladder has changed its orientation and is in a more or less vertical position now).

\subsection{Group C}

Group C consists of particle verbs which can be followed by a pleonastic PP in the accusative or dative. The subgroups of Group $C$ include Verbs of Motion like einsickern 'to soak into', einmarschieren 'to march in', anspülen 'to be washed up' or vorladen 'to subpoena', and Transport Verbs such as aufhängen 'to hang', einschieben 'to insert', einräumen 'to place in' or andocken 'to dock'. Group $\mathrm{C}$ also consists of verbs which express an Inclusion into an Environment, Institution or Abstract Area like eingliedern 'to incorporate', zwischenschalten 'to interpose', aufreihen 'to string' or auffädeln 'to bead'. Another verb group which belongs to Group $\mathrm{C}$ are verbs which express the Localisation of Effects of Action like einprägen 'to impress', einbrennen 'to burn-in', eintätowieren 'to tattoo' or aufdrucken 'to imprint'.

The following example illustrates the semantic effect of the choice of case for the PP for the verbs in Group C:
(9) sickert in die Erde ein soak.3.Sg in Det.Acc soil PART 'soaks into the soil'
(10) sickert in der Erde ein soak.3.Sg in Det.Dat soil PART 'soaks the soil'

Example (9) describes an event where an $\mathrm{X}$ 
(rainwater) undergoes a directed motion during which it enters into the region of the reference object Y (the soil). In (10) the situation is different: $\mathrm{X}$ is already located in the region of $\mathrm{Y}$ and now is in the process of soaking through that region. Figure 3 gives an illustration of the two examples.

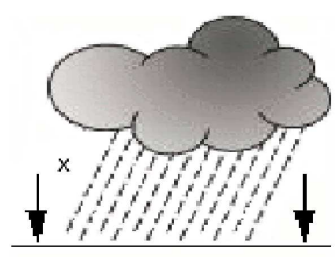

Region of $Y$

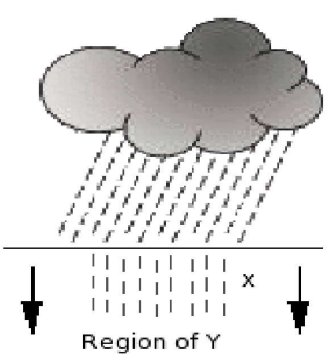

Figure 3: Illustration of examples (9) and (10)
Characteristic for the verbs in Group $\mathrm{C}$ is their directionality reading when going together with a pleonastic PP in the accusative. When they are combined with the dative, the particle still has its directional character, but in contrast to the particle verbs in Group A the directionality does not include an intrusion into another region but can be interpreted as a movement inside of the region given by the reference object.

Summarizing the results we can say that for Group $\mathrm{C}$ the particle can have different functions which influence the choice of case marking for the PPs governed by the verb. If the particle has a nondirectional reading, then only PPs in the dative are allowed. If the particle expresses directional information, then a further analysis is needed: it has to be examined whether the semantics of the particle verb includes the intrusion into a new region specified by the preposition. In this case the PP has to be in the accusative. If the semantics of the verb does not express an intrusion into a new region, then the dative is chosen. Only particle verbs whose semantics allow for a directional and a locative interpretation belong to group $\mathrm{C}$.

In Section 2 we noted that the semantics of the verb particle is equivalent to the semantics of the preposition, and that the PP specifies the implicit reference object of the particle verb. However, this is only true for PPs with accusative case marking. The prepositions in PPs which are in the dative express a locative relation rather than a direction, so their reference object can not be the same as the one implicitly included in the verb particle. On the syntactic level this results in them having a different grammatical function: the accusative PP can be considered as a verb complement, while the dative PP is a free adjunct, modifying the information of the verb particle. Therefore only accusative PPs are "pleonastic".

\section{Description of Particle Verbs with Pleonastic Prepositions in LFG}

This section will show how the framework of Lexical Functional Grammar (LFG) can be used to describe the particular behaviour of particle verbs and pleonastic prepositions.

\subsection{Short Introduction to LFG}

LFG has a layer of representation for constituent structure (c-structure), where surface information is expressed through CFG trees, and a functional layer (f-structure) for expressing grammatical functions such as subject, object and adjunct. In the f-structure each argument of a predicate is assigned a particular grammatical function. This two-level representation is based on the idea that while surface representations may differ considerably between various languages, f-structures tend to be more abstract and invariant representations.

The correspondence between the two layers is many-to-one: different nodes in the c-structure may be associated with the same f-structure component. The c-structure is determined by phrase structure rules as in (11), while the annotation in (12) links the c-structure categories to the corresponding grammatical functions in the f-structure.

(11) $S \rightarrow N P \quad V P$

(12) $\quad(\uparrow$ SUBJ $)=\downarrow \quad \uparrow=\downarrow$

LFG is a non-transformational theory, syntactic phenomena are treated locally through the specification of rules and constraints in the lexicon.

\subsection{Using LFG to Describe Particle Verbs with Pleonastic Prepositions}

The LFG formalisation developed here follows and substantially extends the treatment of particle verbs and prepositional phrases in the LFG grammar for German in (Berman and Frank, 1996) and (Butt, King, Niño and Segond, 1999).

\subsubsection{Berman \& Frank (1996)}

Figure 4 shows the lexical entry for the German particle verb einfahren 'to drive into' as described in (Berman and Frank, 1996). 


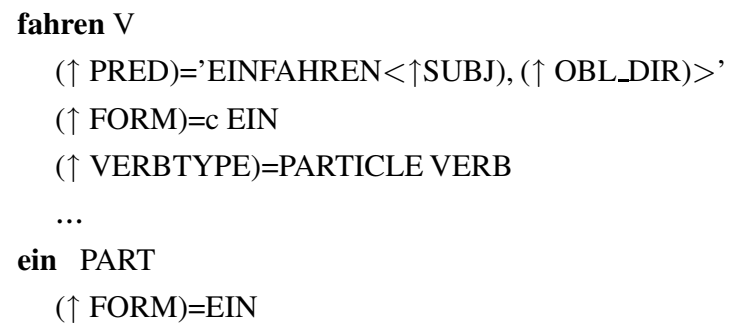

Figure 4: Lexical entry for einfahren 'to drive in' (Berman and Frank, 1996)

The predicate (PRED) shows the argument structure of the verb, while the attribute VERBTYPE explicitly describes the verb as a particle verb. The FORM attribute contains the lexical form of the particle and is formulated as a constraint $(=\mathrm{c})$ to check that the particle is lexically filled. The particle itself has no PRED value of its own but is analysed as part of the complex verb.

German prepositional phrases can either occur as prepositional objects or as adjuncts. According to Berman and Frank (1996) the second group is further subdivided into adjuncts which are subcategorized by the verb and free adjuncts. Accordingly, in the analysis of (Berman and Frank, 1996), each two-way preposition has three lexical entries.

In their analysis, prepositional objects are governed by the verb and have no PRED attribute of their own. The lexical form of the preposition and also its case are determined by the verb. The value of the PCASE attribute is assigned the lexical form of the preposition, while the preposition is not able to subcategorize an object. ${ }^{2}$

As for adjuncts subcategorized by the verb no particular preposition is selected in (Berman and Frank, 1996), but the verb determines the semantic content of the preposition (eg: LOC, DIR). The preposition has its own PRED attribute and subcategorizes an object (Figure 5).

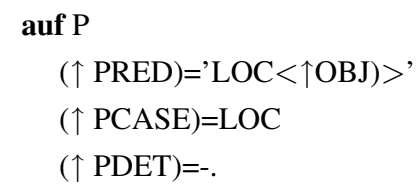

Figure 5: Lexical entry (Berman \& Frank, 1996) for preposition auf 'on' (adjunct subcategorized by the verb)

Free adjuncts on the other hand must have the

\footnotetext{
${ }^{2}$ Prepositional objects are of no concern here, because the paper deals with spatial prepositions which always have a semantic content.
}

semantic content LOC. Like the first type of adjuncts they have their own PRED attribute and subcategorize an object, but their semantic content is defined by the ROLE attribute (Figure 6).

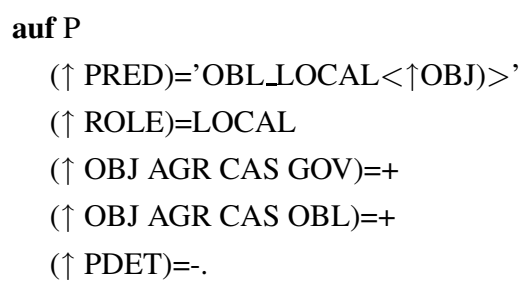

Figure 6: Lexical entry (Berman \& Frank, 1996) for auf' on' (free adjunct)

\subsubsection{Formalisation of Group C Verbs}

We concentrate on the formalisation of the particle verbs in Group $C$ which can either licence a pleonastic PP in the accusative or a PP in the dative. Extending the analysis in (Berman and Frank, 1996) we provide two f-structure configurations, depending on the case of the governed PP.

Figure 7 shows the f-structure for example (9). Here the pleonastic PP in the accusative saturates the argument OBL_DIR subcategorized by the particle verb. Figure 8 gives the f-structure for example (10), where the particle verb combines with a dative PP.

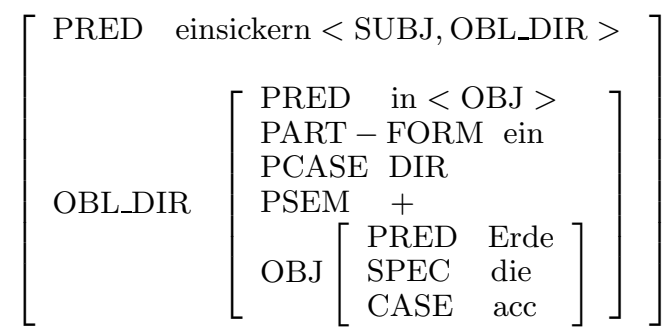

Figure 7: sickert [ PP in die Erde $]_{A C C}$ ein 'soaks into the soil'

In contrast to Figure 7 the dative PP in Figure 8 does not contribute any information to the argument OBL_DIR subcategorized by the verb but is represented in the adjunct set. The verb particle saturizes the OBL_DIR argument, and the PRED attribute of the object of OBL_DIR is assigned the value PRO. This enables the PRED value to behave like a variable which can be unified with any other value as in Figure 8, where both the particle and the pleonastic prepositional phrases add information to OBL_DIR:OBJ:PRED. 


$$
\left[\begin{array}{c}
\text { OBL_DIR }\left[\begin{array}{l}
\text { PRED in }<\text { OBJ }> \\
\text { PART }- \text { FORM ein } \\
\text { PCASE DIR } \\
\text { PSEM + } \\
\text { OBJ }\left[\begin{array}{cc}
\text { PRED } & \text { PRO } \\
\text { CASE } & \text { acc }
\end{array}\right]
\end{array}\right] \\
\text { ADJ }\left\{\left[\begin{array}{l}
\text { PRED in }<\text { OBJ }> \\
\text { PCASE LOC } \\
\text { PSEM + } \\
\text { OBJ }\left[\begin{array}{cc}
\text { PRED Erde } \\
\text { SPEC } \\
\text { CASE }
\end{array}\right]
\end{array}\right]\right\}
\end{array}\right.
$$

Figure 8: sickert [ PP in der Erde $]_{D A T}$ ein 'soaks (through) the soil'

\subsubsection{Lexical Entries and Grammar Rules}

In the f-structure in Figure 7 the pleonastic PP is subcategorized by the particle verb. Figure 9 shows the corresponding lexical entry for the verb. To prevent a locative PP in the dative from filling in the object position of the verb argument the lexical entry specifies that the object has to be assigned accusative case.

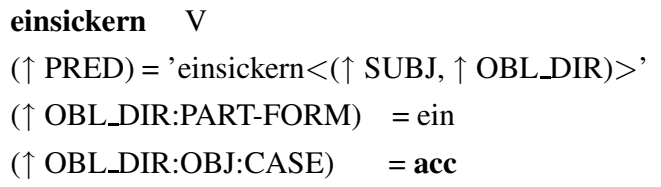

Figure 9: Lexical entry for einsickern 'to soak'

However, as shown in example (4) the pleonastic PP can be omitted. In this case the argument OBL_DIR subcategorized by the particle verb is provided by the particle ein- whose lexical entry is given in Figure 10.

$\begin{array}{ll}\text { ein PART } & \\ (\uparrow \text { PRED }) & =\text { 'in }<(\uparrow \text { OBJ })> \\ (\uparrow \text { PART-FORM }) & =\text { ein } \\ (\uparrow \text { PCASE }) & =\mathrm{DIR} \\ (\uparrow \text { PSEM }) & =+ \\ (\uparrow \text { OBJ PRED }) & =\mathrm{PRO}\end{array}$

Figure 10: Lexical entry for the particle ein

In contrast to (Berman and Frank, 1996), in our representation the particle is assigned the PRED value 'in' in the lexicon. The cause for the divergence between the lexical form of the particle and its PRED value is due to the fact that the particle ein- historically is derived from the preposition in and regarding its semantic features is comparable to the other two-way prepositions where particle and preposition have the same lexical form.

The attributes PSEM and PCASE are added to the representation of the verb particles in Berman and Frank (1996). They are derived from the attribute set for prepositions, indicating the analogy in the semantics of particle and preposition. PSEM always has the value ' + ' for particle verbs formed by spatial prepositions, because they always have a semantic content. The attribute PCASE expresses the directionality in the semantics of the verb particle $((\uparrow$ PCASE $)=$ DIR $)$.

The predicate of the particle licences an object and behaves like a directional preposition. However, the object position is not lexically filled and therefore is assigned the predicate value 'PRO'.

We also want to model the behaviour of the particle verb governing a locative PP in the dative (Figure 8). The lexical entry of the particle verb (Figure 9) explicitly requires accusative case assignment and prevents the locative dative PP from filling in the object position of the verb argument. The locative dative PP is attached to the adjunct set in the grammar rule shown in Figure $11 .^{3}$

$$
\begin{array}{rll}
\mathrm{VP} \rightarrow & \mathrm{V} & \uparrow=\downarrow \\
& \mathrm{PP} * & \downarrow \epsilon(\uparrow \text { ADJ }) \\
& (\downarrow \text { OBJ CASE }) \neq \text { acc } \\
& \text { (PP } & (\uparrow \text { OBL_DIR })=\downarrow) \\
& \text { PART } & (\uparrow \text { OBL_DIR })=\downarrow .
\end{array}
$$

Figure 11: Grammar Rule specifying restrictions on particle verbs with pleonastic PPs

The first PP in the grammar rule models the behaviour of a particle verb combining with one or more locative PPs in the dative. The constraint $(\downarrow$ OBJ CASE $) \neq$ acc ensures that this part of the rule will not be applied to a pleonastic PP with accusative case assignment. ${ }^{4}$

The second PP in the grammar rule captures a pleonastic $\mathrm{PP}$ in the accusative. The restriction that this PP has to be in the accusative is specified in the lexical entry for the particle verb (Figure 10). The last part of the rule expresses that the verb particle PART is also mapped to the OBL_DIR ar-

\footnotetext{
${ }^{3}$ For expository purposes we use a simple VP rather than a topological analysis.

${ }^{4}$ The Kleene $*$ notation indicates zero or more occurences of PP.
} 
gument of the complex verb and so is able to saturate the argument structure of the verb.

The formalisation in Figure 8 and 9 is consistent with the analysis that the particle has an implicit reference object which is identical to the object of a pleonastic PP in the accusative, but not to the object of a dative PP. The formalisation gives an adequate description of the behaviour of particle verbs in Group C, but it does not suppress the licencing of a pleonastic accusative PP for verbs in Group B which combine with locative PPs in the dative only. This problem is solved through the specification of a constraint $(=c)$ in the lexical entries for all particle verbs in Group B (Figure 12).

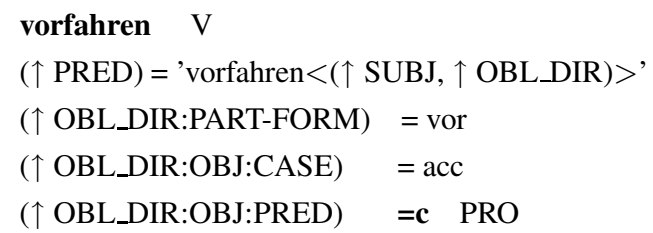

Figure 12: Lexical entry for vorfahren 'to drive up' (Group B)

The constraint checks that the predicate of the object in the OBL_DIR f-structure is instantiated with the value 'PRO'. For all cases where the predicate is lexically realised, the constraint fails and thus the interpretation of pleonastic accusative PPs in the OBL_DIR position for Group B verbs is suppressed.

\section{Conclusions}

The aim of this paper is to explain the behaviour of German particle verbs formed by two-way prepositions and their ability to combine with pleonastic PPs. A classification of particle verbs based on semantic criteria was given, illustrating the restrictions imposed on their behaviour. It was shown that particle verbs occurring only with accusative PPs (Group A) always have a directional reading including the intrusion of the theme referent into a region specified by the relatum. Particle verbs which can not combine with an accusative PP (Group B) either have a static, nondirectional reading or describe a directed movement where the referent already may be present in the region specified by the relatum.

Syntactically this results in the fact that the accusative PP is able to saturate the argument OBL_DIR subcategorized by the particle verbs in Group A. The dative PP functions as an adjunct
(Group B). Here the verb particle saturates the directional OBL_DIR argument required by the verb. Group $\mathrm{C}$ verbs allow both accusative and dative PPs. Only particle verbs governing PPs in the accusative are pleonastic, but the PP either modifies or adds new information to the inherent argument structure of the particle verb and therefore is not suppressed by the verb particle.

Our formalisation describes the behaviour of particle verbs concerning their ability to licence pleonastic PPs. The semantic criteria restricting the behaviour of the particle verbs are embedded into the LFG representation and enable us to model the semantic differences on a syntactic level.

\section{References}

Judith Berman and Anette Frank. 1996. Deutsche und französische Syntax im Formalismus der LFG. Max Niemeyer Verlag, Tübingen.

Joan Bresnan. 2000. Lexical-Functional Syntax. Blackwell.

Miriam Butt, Tracy Holloway King, María-Eugenia Nino and Frédérique Segond. 1999. A Grammar Writer's Cookbook. CSLI Publications, Stanford, California.

Mary Dalrymple. 2001. Syntax and Semantics. Lexical Functional Grammar, volume 34. Academic Press, San Diego, California.

Junji Okamoto. 2002. Particle-Bound Directions in German Particle Verb Constructions. Projektbericht $\mathrm{V}$ : Typological Investigation of Languages and Cultures of the East and West. (Part II).

Susan Olsen. 1998. Semantische und konzeptuelle Aspekte der Partikelverbbildung mit ein-. Stauffenburg, Tübingen.

James Witt. 1998. Kompositionalität und Regularität, In: Olsen, Susan (ed). Semantische und konzeptuelle Aspekte der Partikelverbbildung mit ein-. Stauffenburg, Tübingen.

Dieter Wunderlich. 1983. On the Compositionality of German Prefix Verbs. In: R. Bäuerle, Ch. Schwarze and A. von Stechow (eds.) Meaning, Use and Interpretation of Language. de Gruyter, Berlin. 